\title{
ONE-PARAMETER FAMILIES OF UNIT EQUATIONS
}

\author{
AARON LEVIN
}

\begin{abstract}
We study one-parameter families of $S$-unit equations of the form $f(t) u+$ $g(t) v=h(t)$, where $f, g$, and $h$ are univariate polynomials over a number field, $t$ is an $S$-integer, and $u$ and $v$ are $S$-units. For many possible choices of $f, g$, and $h$, we are able to determine all but finitely many solutions to the corresponding one-parameter family of $S$-unit equations. The results are obtained as consequences of some recent results on integral points on surfaces.
\end{abstract}

\section{Introduction}

An equation of fundamental interest in number theory is the $S$-unit equation

$$
a u+b v=c \quad \text { in } u, v \in \mathcal{O}_{k, S}^{*},
$$

where $k$ is a number field, $a, b, c \in k^{*}, S$ is a finite set of places of $k$ containing the archimedean places, $\mathcal{O}_{k, S}$ is the ring of $S$-integers of $k$, and $\mathcal{O}_{k, S}^{*}$ is the group of $S$-units of $k$. The basic theorem on the $S$-unit equation (1) is

Theorem 1 (Siegel, Mahler). The set of solutions to (1) is finite.

This was proved by Siegel in the case $S$ consists of the set of archimedean places of $k$ and extended by Mahler to arbitrary $S$.

Equation (1) and Theorem 1 have been generalized in at least three distinct directions. First, it is easy to see that Theorem 1 is equivalent to the assertion that, in any affine embedding, $\mathbb{P}^{1} \backslash\{$ three points $\}$ has only finitely many $S$-integral points. Thus, Siegel's theorem on integral points on affine curves may be seen as a generalization of Theorem 1. Second, the hypothesis that $u, v \in \mathcal{O}_{k, S}^{*}$ has been generalized, for instance, by Lang [14] to the assumption that $u, v \in \Gamma$, where $\Gamma$ is a finitely generated subgroup of $\mathbb{C}^{*}$. Third, we can consider $S$-unit equations with more variables and terms. The main theorem in this case, proved independently by Evertse [8] and van der Poorten and Schlickewei [16], is the following.

Theorem 2 (Evertse, van der Poorten and Schlickewei). All but finitely many solutions of the equation

$$
\alpha_{1} u_{1}+\alpha_{2} u_{2}+\ldots+\alpha_{n} u_{n}=\alpha_{n+1} \quad \text { in } u_{1}, \ldots, u_{n} \in \mathcal{O}_{k, S}^{*},
$$

where $\alpha_{1}, \ldots, \alpha_{n+1} \in k^{*}$, satisfy an equation of the form $\sum_{i \in I} \alpha_{i} u_{i}=0$, where $I$ is a subset of $\{0, \ldots, n\}$.

Of course, one can also consider combinations of the above generalizations, such as [10] and [17], which extend Theorem 2 to finitely generated subgroups of $\mathbb{C}^{*}$. We mention also that Theorem 1 has been refined in various ways. There are the effective

Received by the editors January 6, 2006. 
estimates for the heights of solutions to (1) coming from linear forms in logarithms $[11,12]$ and bounds on the number of solutions to (1) depending only on the size of $S$ (and in some versions $[k: \mathbb{Q}])[1,2,7,12]$.

In this article we consider another possible generalization of (1). We study oneparameter families of the two variable $S$-unit equation, namely,

$$
f(t) u+g(t) v=h(t) \quad \text { in } t \in \mathcal{O}_{k, S}, \quad u, v \in \mathcal{O}_{k, S}^{*},
$$

where $f, g$, and $h$ are nonzero polynomials in $k[t]$. In most situations, our requirement that $t \in \mathcal{O}_{k, S}$ can be relaxed to the possibly more natural condition that $t \in k$ (see Lemma 5).

Even for very simple choices of $f, g$, and $h$, Eq. (2) leads to open problems. For instance, taking $f=g=1$ and $h=t^{2}-1$, we obtain the equation

$$
u+v=t^{2}-1 \quad \text { in } t \in \mathcal{O}_{k, S}, \quad u, v \in \mathcal{O}_{k, S}^{*} .
$$

Solving this equation is essentially the same as determining when the sum of three $S$ units is a perfect square. This appears to be a difficult problem. Indeed, determining whether or not there are infinitely many perfect squares in $\mathbb{Z}$ of the form $2^{a}+3^{b}+1$ for positive integers $a$ and $b$ is already an open problem. However, as an example of our results we will show

Theorem 3. For general nonconstant polynomials $f, g, h \in k[t]$ with

$$
\operatorname{deg} f+\operatorname{deg} g=\operatorname{deg} h>2,
$$

the equation

$$
f(t) u+g(t) v=h(t) \quad \text { in } t \in k, \quad u, v \in \mathcal{O}_{k, S}^{*},
$$

has only finitely solutions with $f(t) g(t) h(t) \neq 0$.

By "general" here, we mean that if one parametrizes the polynomials $f, g$, and $h$ in Theorem 3 (fixing their degrees) in the obvious way using the affine space $\mathbb{A}^{2 \operatorname{deg} h+3}$, then we are excluding polynomials $f, g$, and $h$ parametrized by some Zariski-closed subset of $\mathbb{A}^{2 \operatorname{deg} h+3}$ (in principle, the Zariski-closed subset could be explicitly given).

If $t_{0} \in \mathcal{O}_{k, S}$ is not a root of $f, g$, or $h$, then substituting $t=t_{0}$ into (2) gives an $S$-unit equation. Therefore, Theorem 3 gives numerous examples of $S$-unit equations which have no solution. However, since our methods are ineffective, we cannot determine the finitely many values of $t$ for which there are solutions in Theorem 3 , and so we cannot explicitly determine (by our methods) for any given value of $t$ that the corresponding $S$-unit equation has no solutions. Despite our inability to effectively determine the solutions to (3), it is possible to give a bound on the degree of a curve containing, in an appropriate sense, the set of solutions to (3) (see the discussion after Corollary 11).

Our results are proven as consequences of some recent theorems on integral points on surfaces $[6,15]$. These theorems trace their origin to the new proof of Siegel's theorem using the Schmidt subspace theorem given in [3] and developed for surfaces in [5]. The ultimate reliance of our results on the subspace theorem is the reason for their ineffectivity. 


\section{Elementary observations}

Fix nonzero polynomials $f, g$, and $h$. We first discuss an obvious set of solutions to (2). Let $Z(f g h)$ denote the set of zeroes of $f g h$ and suppose that $S$ is large enough such that $Z(f g h) \subset \mathcal{O}_{k, S}$. Equation (2) is not a unit equation for $t \in \mathcal{O}_{k, S}$ exactly when $t \in Z(f g h)$. For these values of $t$, the set of solutions to (2) is easily described. For instance, if $f\left(t_{0}\right)=0$ and $g\left(t_{0}\right) \neq 0$, then the set of solutions to (2) with $t=t_{0}$ is given by $u \in \mathcal{O}_{k, S}^{*}$ and $v=\frac{h\left(t_{0}\right)}{g\left(t_{0}\right)}$, assuming that $\frac{h\left(t_{0}\right)}{g\left(t_{0}\right)} \in \mathcal{O}_{k, S}^{*}$ (otherwise, there are no solutions with $\left.t=t_{0}\right)$. Thus, we will call any solution with $t \in Z(f g h)$ a trivial solution and any solution with $t \notin Z(f g h)$ a nontrivial solution.

In the rest of the paper, we will always make the assumption that $f$ and $g$ do not have a common zero. We now show that there is no loss of generality in doing this.

Lemma 4. Let $f, g, h \in k[t]$ be nonzero polynomials. Then there exist polynomials $f^{\prime}, g^{\prime}, h^{\prime} \in k[t]$ such that $f^{\prime}$ and $g^{\prime}$ do not have a common zero and such that, for large enough $S$, there is a natural inclusion of the set of nontrivial solutions to

$$
f(t) u+g(t) v=h(t) \quad \text { in } t \in \mathcal{O}_{k, S}, \quad u, v \in \mathcal{O}_{k, S}^{*},
$$

into the set of nontrivial solutions to

$$
f^{\prime}\left(t^{\prime}\right) u^{\prime}+g^{\prime}\left(t^{\prime}\right) v^{\prime}=h^{\prime}\left(t^{\prime}\right) \quad \text { in } t^{\prime} \in \mathcal{O}_{k, S}, \quad u^{\prime}, v^{\prime} \in \mathcal{O}_{k, S}^{*} .
$$

Proof. We easily reduce to the case where $f, g$, and $h$ do not all have a common zero. Let $d \in k[t]$ be such that $f^{\prime}=f / d$ and $g^{\prime}=g / d$ do not have a common zero and $f^{\prime}, g^{\prime} \in \mathcal{O}_{k, S}[t]$. For any nontrivial solution $(t, u, v)$ satisfying (4) we have $f^{\prime}(t) u+g^{\prime}(t) v=h(t) / d(t) \in \mathcal{O}_{k, S}$. It follows from the fact that $h$ and $d$ do not have a common zero that, after enlarging $S, d(t) \in \mathcal{O}_{k, S}^{*}$ for any nontrivial solution $(t, u, v)$ satisfying (4). Therefore, if $(t, u, v)$ is a nontrivial solution to (4) then $\left(t^{\prime}, u^{\prime}, v^{\prime}\right)=$ $(t, u d(t), v d(t))$ is a nontrivial solution to (5), where we have set $h^{\prime}=h$.

Finally, in most situations the restriction that $t$ is an $S$-integer in (2) is unnecessary.

Lemma 5. Suppose that the largest degree among $f, g$, and $h$ is uniquely attained among $f, g$, and $h$. Then for large enough $S$, the set of solutions to

$$
f(t) u+g(t) v=h(t) \quad \text { in } t \in k, u, v \in \mathcal{O}_{k, S}^{*},
$$

is the same as the set of solutions to (2).

Proof. Clearly, we can assume that $f, g, h \in \mathcal{O}_{k}[t]$. Suppose that $S$ is large enough such that the leading coefficients of $f, g$, and $h$ are all $S$-units. Let $u, v \in \mathcal{O}_{k, S}^{*}$. Then by our assumption on the degrees of $f, g$, and $h$, it follows that the leading coefficient of $f(t) u+g(t) v-h(t)$ (as a polynomial in $t$ ) is an $S$-unit. Therefore, if $(t, u, v)$ is a solution to (6) then $t$ must be an $S$-integer.

\section{Integral points on certain affine surfaces}

We start with a definition of integral points for affine varieties.

Definition 6. Let $V$ be an affine variety defined over a number field $k$. Let $S$ be a finite set of places of $k$ containing the archimedean places. We define a set $R \subset V(k)$ to be a set of $S$-integral points on $V$ if there exists an affine embedding $\phi: V \hookrightarrow \mathbb{A}^{n}$ such that $\phi(R) \subset \mathbb{A}^{n}\left(\mathcal{O}_{k, S}\right)$. 
The solutions to (2) are intimately related to integral points on certain affine surfaces. For our purposes, it will be most convenient to view the affine surfaces of interest as subsets of $\mathbb{P}^{1} \times \mathbb{P}^{1}$.

Theorem 7. Let $f, g, h \in k[t]$ be nonzero polynomials and let

$$
T=\left\{(t, u, v) \in \mathcal{O}_{k, S} \times \mathcal{O}_{k, S}^{*} \times \mathcal{O}_{k, S}^{*} \mid f(t) u+g(t) v=h(t)\right\}
$$

be the set of solutions to (2). Suppose that $f$ and $g$ do not have a common zero. Let $\tilde{f}, \tilde{g} \in k[t]$ be such that $f \tilde{g}+g \tilde{f}=h$. Consider $\mathbb{P}^{1} \times \mathbb{P}^{1}$ with coordinates $\left(x_{1}, y_{1}\right) \times$ $\left(x_{2}, y_{2}\right)$. Let $Z$ be the closed subset of $\mathbb{P}^{1} \times \mathbb{P}^{1}$ that is the union of the sets defined by the four equations (appropriately clearing denominators in the last two equations)

$$
\begin{aligned}
y_{1} & =0, \\
y_{2} & =0, \\
x_{1} f\left(\frac{x_{2}}{y_{2}}\right)-y_{1} \tilde{f}\left(\frac{x_{2}}{y_{2}}\right) & =0, \\
x_{1} g\left(\frac{x_{2}}{y_{2}}\right)+y_{1} \tilde{g}\left(\frac{x_{2}}{y_{2}}\right) & =0 .
\end{aligned}
$$

Let $R \subset \mathbb{P}^{1} \times \mathbb{P}^{1} \backslash Z$ be the set

$$
\begin{aligned}
R=\{(\tilde{f}(t)-v, f(t)) \times(t, 1) \mid & (t, u, v) \in T, f(t) \neq 0\} \cup \\
& \{(u-\tilde{g}(t), g(t)) \times(t, 1) \mid(t, u, v) \in T, g(t) \neq 0\} .
\end{aligned}
$$

Then $R$ is a set of $S$-integral points on $\mathbb{P}^{1} \times \mathbb{P}^{1} \backslash Z$.

Proof. Multiplying everything by a scalar, we may assume that $f, g, h \in \mathcal{O}_{k}[t]$. We first show that there exists a constant $c \in k^{*}$ such that for all $(t, u, v) \in T$,

$$
\frac{c(\tilde{f}(t)-v)}{f(t)} \in \mathcal{O}_{k, S} \text { if } f(t) \neq 0, \quad \frac{c(u-\tilde{g}(t))}{g(t)} \in \mathcal{O}_{k, S} \text { if } g(t) \neq 0 .
$$

By possibly making $c$ larger, it clearly suffices to prove this for all but finitely many values of $t$. So we will ignore values of $t$ for which $f(t)=0$ or $g(t)=0$. In this case, it follows from (2) and the definitions of $\tilde{f}$ and $\tilde{g}$ that

$$
\frac{\tilde{f}(t)-v}{f(t)}=\frac{u-\tilde{g}(t)}{g(t)} .
$$

Since $f$ and $g$ do not have a common zero, there exist polynomials $p_{1}, p_{2} \in \mathcal{O}_{k}[t]$ such that $f p_{1}+g p_{2}=a$, where $a \in \mathcal{O}_{k}$ is a constant. Let $b_{1}, b_{2} \in \mathcal{O}_{k}$ be nonzero elements such that $b_{1} \tilde{f}$ and $b_{2} \tilde{g}$ have integral coefficients. Then it follows from (9) and the fact that $t, u, v \in \mathcal{O}_{k, S}$ that we can take $c=a b_{1} b_{2}$ in (8) if $f(t) g(t) \neq 0$.

Since $y_{1} y_{2} \neq 0$ on $V=\mathbb{P}^{1} \times \mathbb{P}^{1} \backslash Z$, let $x_{1}^{\prime}=x_{1} / y_{1}$ and $x_{2}^{\prime}=x_{2} / y_{2}$ be coordinates on $V$. Then every regular function on $V$ may we be written as $p\left(x_{1}^{\prime}, x_{2}^{\prime}\right) /\left(\left(x_{1}^{\prime} f\left(x_{2}^{\prime}\right)-\right.\right.$ $\left.\left.\tilde{f}\left(x_{2}^{\prime}\right)\right)^{m}\left(x_{1}^{\prime} g\left(x_{2}^{\prime}\right)+\tilde{g}\left(x_{2}^{\prime}\right)\right)^{n}\right)$, where $p$ is a polynomial in two variables and $m$ and $n$ are integers. A simple calculation shows that for $(t, u, v) \in T$, if $x_{1}^{\prime}=(\tilde{f}(t)-v) / f(t)$ or $x_{1}^{\prime}=(u-\tilde{g}(t)) / g(t)$, and $x_{2}^{\prime}=t$, then

$$
\begin{aligned}
& x_{1}^{\prime} f\left(x_{2}^{\prime}\right)-\tilde{f}\left(x_{2}^{\prime}\right)=-v, \\
& x_{1}^{\prime} g\left(x_{2}^{\prime}\right)+\tilde{g}\left(x_{2}^{\prime}\right)=u .
\end{aligned}
$$


For these values of $x_{1}^{\prime}$ and $x_{2}^{\prime}$, it follows from (8) that there exists a constant $d \in k^{*}$ such that $d p\left(x_{1}^{\prime}, x_{2}^{\prime}\right) \in \mathcal{O}_{k, S}$. Therefore, for any regular function $\psi$ on $V$ we see that there exists a constant $d \in k^{*}$ such that $d \psi(R) \subset \mathcal{O}_{k, S}$. Note also that $V$ is affine. Thus, after multiplying the coordinate functions by suitable constants, for any affine embedding $\phi: V \hookrightarrow \mathbb{A}^{N}$ we have $\phi(R) \subset \mathbb{A}^{N}\left(\mathcal{O}_{k, S}\right)$.

So the problem of determining solutions to (2) is now reduced to the study of $S$ integral points on certain affine surfaces. When there does not exist a Zariski-dense set of $S$-integral points on such a surface, we can parametrize the solutions to the corresponding one-parameter $S$-unit equation.

Theorem 8. Let $f, g, h$, and $Z \subset \mathbb{P}^{1} \times \mathbb{P}^{1}$ be as in Theorem \%. Suppose that there does not exist a Zariski-dense set of $S$-integral points on $\mathbb{P}^{1} \times \mathbb{P}^{1} \backslash Z$. Then there exist finitely many quintuples $\left(z_{i}, a_{i}, b_{i}, p_{i}, q_{i}\right), z_{i} \in k\left[t, \frac{1}{t}\right], a_{i}, b_{i} \in k, p_{i}, q_{i} \in \mathbb{Z}$, with

$$
a_{i} f\left(z_{i}(t)\right) t^{p_{i}}+b_{i} g\left(z_{i}(t)\right) t^{q_{i}}=h\left(z_{i}(t)\right)
$$

for $i=1, \ldots, j$, such that all solutions to (2) are parametrized by

$$
t=z_{i}(s), u=a_{i} s^{p_{i}}, v=b_{i} s^{q_{i}}, s \in k
$$

for $i=1, \ldots, j$.

This follows easily from Siegel's theorem.

Proof. Let $R$ be as in (7). Then by Theorem $2, R$ is a set of $S$-integral points on $\mathbb{P}^{1} \times \mathbb{P}^{1} \backslash Z$. By hypothesis, $R$ is not Zariski-dense. Let $C_{i}, i=1, \ldots, j$, be the onedimensional irreducible components of the Zariski-closure of $R$ in $\mathbb{P}^{1} \times \mathbb{P}^{1}$. By Siegel's theorem, $C_{i}$ is a rational curve defined over $k$, and if $\phi_{i}: \mathbb{P}^{1} \rightarrow C_{i} \rightarrow \mathbb{P}^{1} \times \mathbb{P}^{1}$ is the normalization map composed with the inclusion map of $C_{i}$, then $\# \phi_{i}^{-1}\left(Z \cap C_{i}\right) \leq 2$. After an automorphism of $\mathbb{P}^{1}$, we can assume that $\phi_{i}^{-1}\left(Z \cap C_{i}\right) \subset\{0, \infty\} \subset \mathbb{P}^{1}$. Let $\phi_{i}(t)=\left(y_{i}(t), 1\right) \times\left(z_{i}(t), 1\right)$ in affine coordinates on $\mathbb{P}^{1}$. By the definition of $Z$ and our assumption that $\phi_{i}^{-1}\left(Z \cap C_{i}\right) \subset\{0, \infty\}$, we then have $y_{i}, z_{i} \in k\left[t, \frac{1}{t}\right]$ and

$$
\begin{gathered}
y_{i}(t) f\left(z_{i}(t)\right)-\tilde{f}\left(z_{i}(t)\right)=-b_{i} t^{q_{i}}, \\
y_{i}(t) g\left(z_{i}(t)\right)+\tilde{g}\left(z_{i}(t)\right)=a_{i} t^{p_{i}}
\end{gathered}
$$

for some $a_{i}, b_{i} \in k$ and some integers $p_{i}, q_{i} \in \mathbb{Z}$. Now easy calculations and the definition of $R$ show that (10) holds and that all but finitely many solutions to (2) are parametrized by (11) for $i=1, \ldots, j$. The finitely many remaining solutions can be covered in the theorem by taking, for some $i^{\prime}, z_{i^{\prime}}(s)$ constant and $p_{i^{\prime}}=q_{i^{\prime}}=0$ with appropriate $a_{i^{\prime}}, b_{i^{\prime}} \in k$.

We define a set of curves on a surface to be in general position if the intersection of any three of the curves is empty. Recall also that a curve $C$ on $\mathbb{P}^{1} \times \mathbb{P}^{1}$ is said to be of type $(a, b)$ if it is defined by a bihomogeneous equation of bidegree $(a, b)$. We will need the following theorem on integral points from [15].

Theorem 9. Let $Z_{1}, Z_{2}, Z_{3}, Z_{4} \subset \mathbb{P}^{1} \times \mathbb{P}^{1}$ be curves in general position of types $(0,1),(1,0),(1, m)$, and $(1, n)$, respectively, with $m, n>0$. Then there exists a proper Zariski-closed subset $Y \subset \mathbb{P}^{1} \times \mathbb{P}^{1}$, independent of $k$ and $S$, such that for any set $R$ of $S$-integral points on $\mathbb{P}^{1} \times \mathbb{P}^{1} \backslash \cup_{i=1}^{4} Z_{i}$ the set $R \backslash Y$ is finite. 
Remark. Using a quantitative version of Schmidt's subspace theorem due to Evertse [9], and the method of proof in [15], it is possible to show that any set $R$ in Theorem 9 is contained in a curve $C \subset \mathbb{P}^{1} \times \mathbb{P}^{1}$ of type $(a, b)$, where $a, b \leq N\left(\phi, Z_{3}, Z_{4},[k: \mathbb{Q}],|S|\right)$. Here $N\left(\phi, Z_{3}, Z_{4},[k: \mathbb{Q}],|S|\right)$ is an explicitly computable function which depends only on the embedding $\phi$ from Definition $6, Z_{3}$ and $Z_{4}$ (specifically, $m, n$, and the heights of the equations defining $Z_{3}$ and $Z_{4}$ ), the degree $[k: \mathbb{Q}]$, and the number of places in $S$. A more complicated and detailed calculation of this sort is carried out in [4], where Corvaja and Zannier use their proof of Siegel's theorem combined with Evertse's result to obtain new bounds for the number of integral points on affine curves.

More generally, Theorem 9 holds with $Z_{3}$ and $Z_{4}$ of types $(a, b)$ and $(c, d)$, respectively, with $a, b, c, d>0$. We now give some more information on the exceptional set $Y$ in Theorem 9 in the case that $Z_{1}, Z_{2}, Z_{3}$, and $Z_{4}$ meet transversally (i.e., if $P \in Z_{i} \cap Z_{j}, i \neq j$, then the local equations $f, g$ of $Z_{i}, Z_{j}$ at $P$ generate the maximal ideal of $\left.\mathcal{O}_{P, \mathbb{P}^{1} \times \mathbb{P}^{1}}\right)$. It is easy to see that every curve on $\mathbb{P}^{1} \times \mathbb{P}^{1}$ intersects $Z=\cup_{i=1}^{4} Z_{i}$ in at least two points. So by Siegel's theorem, every irreducible curve $C$ in (a minimal) $Y$ intersects $Z$ in exactly two points $P$ and $Q$ ignoring multiplicities. We denote the intersection number of two curves $D$ and $E$ on a surface by D.E.

Theorem 10. Suppose that $Z_{1}, Z_{2}, Z_{3}$, and $Z_{4}$ are in general position and meet transversally. Then the set $Y$ (minimally chosen) consists of the following types of irreducible curves $C$ :

(i). $C$ is of type $(0,1)$.

(ii). $C$ is of type $(1,0)$.

(iii). $C$ is of type $(1,1)$ and $P \in Z_{1} \cap Z_{3}, Q \in Z_{2} \cap Z_{4}$ (up to switching $P$ and $Q$ or $Z_{3}$ and $\left.Z_{4}\right)$, where $\{P, Q\}=C \cap Z$.

Furthermore, if $m+n>2$, then for general $Z_{3}$ and $Z_{4}$ of types $(1, m)$ and $(1, n)$, respectively, $Y$ consists only of $(0,1)$ curves.

Proof. By Siegel's theorem, $C$ must be a rational curve and, as noted earlier, $C$ must intersect $Z$ in exactly two points $P$ and $Q$. Furthermore, if $\pi: \mathbb{P}^{1} \rightarrow C$ is the normalization of $C$, then $\pi^{-1}(P)$ and $\pi^{-1}(Q)$ must consist of exactly one point each. Suppose that $C$ is of type $(a, b)$ with $a, b>0$. Then $C$ intersects each of $Z_{1}, Z_{2}, Z_{3}$, and $Z_{4}$ in at least one point. Therefore, up to switching $P$ and $Q$ or $Z_{3}$ and $Z_{4}$, since the curves in $Z$ are in general position we must have either $P \in Z_{1} \cap Z_{2}, Q \in Z_{3} \cap Z_{4}$ or $P \in Z_{1} \cap Z_{3}, Q \in Z_{2} \cap Z_{4}$.

Suppose that $P \in Z_{1} \cap Z_{2}$ and $Q \in Z_{3} \cap Z_{4}$. Recall the intersection formula [13, Ch. V:Ex. 3.2]

$$
D . E=\sum \mu_{P^{\prime}}(D) \mu_{P^{\prime}}(E)
$$

for curves $D$ and $E$ on $\mathbb{P}^{1} \times \mathbb{P}^{1}$ without shared components, where $\mu_{P^{\prime}}(D)$ and $\mu_{P^{\prime}}(E)$ denote the multiplicity of the point $P^{\prime}$ on $D$ and $E$, respectively, and the sum is taken over all infinitely near points $P^{\prime}$ of $\mathbb{P}^{1} \times \mathbb{P}^{1}$. Let $\phi: X \rightarrow \mathbb{P}^{1} \times \mathbb{P}^{1}$ be the map obtained by blowing up $\mathbb{P}^{1} \times \mathbb{P}^{1}$ at $Q$. Let $Z_{3}^{\prime}, Z_{4}^{\prime}$, and $C^{\prime}$ denote the strict transforms of $Z_{3}$, $Z_{4}$, and $C$, respectively, in $X$. By transversality, $Z_{3}^{\prime}$ and $Z_{4}^{\prime}$ do not meet above $Q$. Since $C$ intersects $Z_{3}$ and $Z_{4}$ only at $Q$ and $\pi^{-1}(Q)$ consists of exactly one point, it follows that $C^{\prime}$ can intersect at most one of $Z_{3}^{\prime}$ and $Z_{4}^{\prime}$. Therefore, if $C^{\prime}$ does not intersect, say, $Z_{3}^{\prime}$, then applying (12) to $C$ and $Z_{3}$, we see that we must have 
$\mu_{Q}(C)=C \cdot Z_{3}=a m+b \geq a+b$. But it is easy to see that an irreducible curve of type $(a, b)$ with $a, b>0$ cannot have a point of multiplicity $\geq a+b$. Thus, we arrive at a contradiction in this case.

Now suppose that $P \in Z_{1} \cap Z_{3}, Q \in Z_{2} \cap Z_{4}$. The same argument as before, making use of the transversality assumptions, shows that $\mu_{P}(C)=a$ and $\mu_{Q}(C)=b$. It is an easily verified fact that given any two points $P^{\prime}, Q^{\prime} \in \mathbb{P}^{1} \times \mathbb{P}^{1}$ there is a (unique) $(1,1)$ curve passing through $P^{\prime}$ and $Q^{\prime}$ with a given tangent direction at $P^{\prime}$. Let $D$ be a curve of type $(1,1)$ passing through $P$ and $Q$ and tangent to $C$ at $P$. If $D \neq C$, then by construction $C . D>a+b$, a contradiction. Therefore we must have $C=D$, and so $C$ must be a curve of type $(1,1)$.

Finally, if $m+n>2$, it is not hard to see that for $Y$ to contain curves other than curves of type $(0,1)$, the curves in $Z$ must be in special position, definable by algebraic relations. We leave the details to the reader.

As a consequence of Theorems 9 and 10 we obtain

Corollary 11. Let $f, g, h \in k[t]$ be nonconstant polynomials such that $f$ and $g$ do not have a common zero and $\operatorname{deg} f+\operatorname{deg} g=\operatorname{deg} h$. Then all but finitely many solutions to

$$
f(t) u+g(t) v=h(t) \quad \text { in } t \in k, \quad u, v \in \mathcal{O}_{k, S}^{*},
$$

are parametrized by a finite number of families, independent of $k$ and $S$, of the form

$$
t=z(s), u=a s^{p}, v=b s^{q}, \quad s \in k,
$$

where $z \in k[t], a, b \in k, p, q \in \mathbb{Z}$, and

$$
a f(z(t)) t^{p}+b g(z(t)) t^{q}=h(z(t)) .
$$

If in addition all of the roots of $f, g$, and $h$ occur with multiplicity one, then all of the parametrizations (14) can be chosen with z linear.

Proof. Let $m=\operatorname{deg} f$ and $n=\operatorname{deg} g$. First note that there exist $\tilde{f}, \tilde{g} \in k[t]$ with $\operatorname{deg} \tilde{f} \leq m$ and $\operatorname{deg} \tilde{g} \leq n$ such that $f \tilde{g}+g \tilde{f}=h$. To see this, let $P_{i}$ denote the vector space of polynomials over $k$ of degree at most $i$ and consider the map $P_{n} \oplus P_{m} \rightarrow P_{m+n}$ given by $x \oplus y \mapsto f x+g y$. The kernel is one-dimensional, generated by $(-g) \oplus f$ (since $f$ and $g$ do not have a common zero), and so by counting dimensions we see that the map is surjective. Therefore, by Theorem 7 with this $\tilde{f}$ and $\tilde{g}$, we see that solutions to (13) give rise to a set of $S$-integral points on $\mathbb{P}^{1} \times \mathbb{P}^{1} \backslash Z$, where $Z=\cup_{i=1}^{4} Z_{i}$ and $Z_{1}, Z_{2}$, $Z_{3}$, and $Z_{4}$ are of types $(1,0),(0,1),(1, m)$, and $(1, n)$, respectively. Furthermore, it is easy to see that $\operatorname{deg} f+\operatorname{deg} g=\operatorname{deg} h$ implies that $Z_{1}, Z_{2}, Z_{3}$, and $Z_{4}$ are in general position (in fact, the other direction also holds). The first part of the corollary then follows from Theorems 8 and 9, except for the extra assertion that $z$ can be chosen in $k[t]$ rather than $k[t, 1 / t]$. This last assertion follows from the fact that the $Z_{i}$ 's are in general position, and so each $C_{i}$ in the proof of Theorem 8 intersects $Z_{1}$ and $Z_{2}$ in only one point each. Finally, the condition that all of the roots of $f, g$, and $h$ occur with multiplicity one (and $\operatorname{deg} f+\operatorname{deg} g=\operatorname{deg} h$ ) is readily seen to be equivalent to $Z_{1}, Z_{2}, Z_{3}$, and $Z_{4}$ being in general position and meeting transversally. Thus, we may use Theorem 10. Since the parametrization (14) corresponding to a curve $C$ of type $(c, d)$ has $\operatorname{deg} z=c$, by Theorem 10, $z$ can be chosen to be linear. 
Theorem 3 from the introduction is similarly a consequence of the last statement of Theorem 10. We merely note that a $(0,1)$ curve in $Y$ corresponds to a trivial set of solutions to (3). It is natural to try to determine how the cardinality of the set of nontrivial solutions to (3) in Theorem 3 depends on the polynomials $f, g$, and $h$ and the set of places $S$. Viewing the nontrivial solutions of (3) as a subset $R \subset \mathbb{P}^{1} \times \mathbb{P}^{1}$ as in Theorem 7, using the remark following Theorem 9 it can be shown that $R$ is contained in a curve $C \subset \mathbb{P}^{1} \times \mathbb{P}^{1}$ of type $(a, b)$ with $a, b \leq N(f, g, h,[k: \mathbb{Q}],|S|)$. Here $N(f, g, h,[k: \mathbb{Q}],|S|)$ is an explicitly computable function that depends only on the degrees and heights of the polynomials $f, g$, and $h$, the degree $[k: \mathbb{Q}]$, and the number of places in $S$. Of course, in the situation of Theorem $3, R$ is in fact a finite set, and it seems quite plausible that $R$ is itself bounded in cardinality by a number depending only on the heights of $f, g$, and $h$, the degree $[k: \mathbb{Q}]$, and the number of places in $S$.

As an example of Corollary 11, we explicitly work out what happens when $f$ and $g$ are linear and $h$ is quadratic.

Corollary 12. Let $L_{1}=a_{1} t+a_{0}$ and $L_{2}=b_{1} t+b_{0}$ with $L_{1}, L_{2} \in k[t], L_{1} / L_{2}$ nonconstant, and $a_{1}, b_{1} \neq 0$. Let $Q=c_{2} t^{2}+c_{1} t+c_{0} \in k[t]$ with $c_{2} \neq 0$. Consider the equation

$$
L_{1}(t) u+L_{2}(t) v=Q(t) \quad \text { in } t \in k, \quad u, v \in \mathcal{O}_{k, S}^{*}
$$

Let $r_{1}$ and $r_{2}$ be the roots of $Q$. Then there exist the following four families of (potential) solutions to (15):

$$
t=\frac{\left(a_{1} b_{0}-a_{0} b_{1}\right) \eta}{c_{2}\left(b_{1} r_{1}+b_{0}\right)}+r_{2}, \quad u=\eta, \quad v=-\frac{\left(a_{1} r_{1}+a_{0}\right) \eta}{b_{1} r_{1}+b_{0}}, \quad \eta \in \mathcal{O}_{k, S}^{*},
$$

$$
t=\frac{\left(a_{1} b_{0}-a_{0} b_{1}\right) \eta}{c_{2}\left(b_{1} r_{2}+b_{0}\right)}+r_{1}, \quad u=\eta, \quad v=-\frac{\left(a_{1} r_{2}+a_{0}\right) \eta}{b_{1} r_{2}+b_{0}}, \quad \eta \in \mathcal{O}_{k, S}^{*},
$$

$$
t=\frac{a_{1} \eta}{c_{2}}+\frac{a_{1} b_{1} c_{0}-a_{1} b_{0} c_{1}+a_{0} b_{0} c_{2}}{c_{2}\left(a_{1} b_{0}-a_{0} b_{1}\right)}, u=\eta, v=\frac{a_{1}^{2} c_{0}-a_{0} a_{1} c_{1}+a_{0}^{2} c_{2}}{a_{1}\left(a_{1} b_{0}-a_{0} b_{1}\right)}, \eta \in \mathcal{O}_{k, S}^{*},
$$

$$
t=\frac{b_{1} \eta}{c_{2}}+\frac{a_{1} b_{1} c_{0}-a_{0} b_{1} c_{1}+a_{0} b_{0} c_{2}}{c_{2}\left(a_{0} b_{1}-a_{1} b_{0}\right)}, u=\frac{b_{1}^{2} c_{0}-b_{0} b_{1} c_{1}+b_{0}^{2} c_{2}}{b_{1}\left(a_{0} b_{1}-a_{1} b_{0}\right)}, v=\eta, \eta \in \mathcal{O}_{k, S}^{*} .
$$

All but finitely many solutions to (15) are given as follows:

(a). If $Q$ is not a perfect square and not of the form $\alpha L_{1} L_{2}+\beta, \alpha, \beta \in k$, then all but finitely many nontrivial solutions to (15) are contained in (16)-(19).

(b). If $Q$ is not a perfect square and $Q=\alpha L_{1} L_{2}+\beta$ for some $\alpha, \beta \in k$, then all but finitely many nontrivial solutions to (15) are contained in (16)-(19) and 
the following two families:

$$
\begin{array}{lll}
t=\frac{a_{1} \eta}{c_{2}}-\frac{b_{0}}{b_{1}}, & u=\eta, \quad v=\frac{c_{2}\left(a_{1} b_{1} c_{0}-a_{0} b_{0} c_{2}\right)}{a_{1}^{2} b_{1}^{2} \eta}, & \eta \in \mathcal{O}_{k, S}^{*} \\
t=\frac{b_{1} \eta}{c_{2}}-\frac{a_{0}}{a_{1}}, & u=\frac{c_{2}\left(a_{1} b_{1} c_{0}-a_{0} b_{0} c_{2}\right)}{a_{1}^{2} b_{1}^{2} \eta}, \quad v=\eta, & \eta \in \mathcal{O}_{k, S}^{*} .
\end{array}
$$

(c). If $Q$ is a perfect square with double root $r=r_{1}=r_{2}$ and $Q$ is not of the form $\alpha L_{1} L_{2}+\beta, \alpha, \beta \in k$, then all but finitely many nontrivial solutions to (15) are contained in (16)-(19) and the following family:

$$
t=\eta \sqrt{\frac{a_{0} b_{1}-a_{1} b_{0}}{b_{1} c_{2}}}+r, \quad u=\eta^{2}, \quad v=-\frac{a_{1} \eta^{2}}{b_{1}}, \quad \eta \in \mathcal{O}_{k, S}^{*} .
$$

(d). If $Q$ is a perfect square with double root $r=r_{1}=r_{2}$ and $Q=\alpha L_{1} L_{2}+\beta$ for some $\alpha, \beta \in k$, then all but finitely many nontrivial solutions to (15) are contained in (16)-(22).

Proof. As in the proof of Corollary 11, the solutions to (15) give rise to integral points on $\mathbb{P}^{1} \times \mathbb{P}^{1} \backslash Z$, where $Z=\cup_{i=1}^{4} Z_{i}$ and $Z_{1}, Z_{2}, Z_{3}$, and $Z_{4}$ are curves in general position of types $(0,1),(1,0),(1,1)$, and $(1,1)$, respectively. The four families of solutions (16)-(19) correspond to the four curves of type $(1,0)$ that intersect $Z$ in exactly two points. If $Q$ is not a perfect square, the curves $Z_{1}, Z_{2}, Z_{3}$, and $Z_{4}$ meet transversally. Thus, by Theorem 10 , the only other infinite families of nontrivial solutions in cases (a) and (b) arise from curves of type $(1,1)$ as in (iii) of Theorem 10. It is easily verified that such curves exist if and only if $Q$ is of the form $Q=\alpha L_{1} L_{2}+\beta$ for some $\alpha, \beta \in k$, in which case there are two curves of type $(1,1)$ intersecting $Z$ in exactly two points, corresponding to the two families of solutions (20) and (21). This proves parts (a) and (b).

When $Q$ is a perfect square, the curves $Z_{3}$ and $Z_{4}$ intersect in only one point (and hence Theorem 10 does not apply). In this case we give an analysis of the exceptional set $Y$ in Theorem 9 similar to the analysis given in Theorem 10. Let $\left\{P_{i, j}\right\}=Z_{i} \cap Z_{j}, 1 \leq i<j \leq 4$. Let $C$ be a curve of type $(a, b), a, b>0$, that is a necessary component of the exceptional set $Y$ in Theorem 9. Then $C \cap Z=\left\{P_{i, j}, P_{k, l}\right\}$ with $\{i, j, k, l\}=\{1,2,3,4\}$. For $i<j,(i, j) \neq(3,4)$, the curves $Z_{i}$ and $Z_{j}$ intersect transversally at $P_{i, j}$. Therefore the proof of Theorem 10 shows that if $P_{3,4} \notin C$, then $C$ must be a curve of type $(1,1)$ as in (iii) of Theorem 10. As mentioned previously, such a curve $C$ exists if and only if $Q$ is of the form $Q=\alpha L_{1} L_{2}+\beta$ for some $\alpha, \beta \in k$. Now suppose that $C \cap Z=\left\{P_{1,2}, P_{3,4}\right\}$. Let $X^{\prime}$ denote the blowup of $\mathbb{P}^{1} \times \mathbb{P}^{1}$ at $P_{3,4}$ and let $P_{3,4}^{\prime}$ be the (unique) point of intersection of the strict transforms of $Z_{3}$ and $Z_{4}$ on $X^{\prime}$. Since the strict transforms of $Z_{3}$ and $Z_{4}$ meet transversally at $P_{3,4}^{\prime}$, an argument as in the proof of Theorem 10 shows that $\mu_{P_{3,4}}(C)+\mu_{P_{3,4}^{\prime}}(C)=\min \left\{C . Z_{3}, C . Z_{4}\right\}=a+b$. Let $C^{\prime}$ be a curve of type $(1,1)$ passing through $P_{1,2}$ and tangent to $Z_{3}$ (and $Z_{4}$ ) at $P_{3,4}$. Then if $C \neq C^{\prime}$, by (12) we obtain $C^{\prime} . C \geq a+b+1$, which is impossible. Therefore we must have $C=C^{\prime}$. The curve $C^{\prime}$ corresponds to the family of solutions (22). Parts (c) and (d) of the theorem now follow.

We can also prove a result in one case where $\operatorname{deg} f+\operatorname{deg} g \neq \operatorname{deg} h$. We need the following special case of a result from [6]. 
Theorem 13 (Corvaja, Zannier). Let $Z_{1}, Z_{2}, Z_{3}, Z_{4} \subset \mathbb{P}^{1} \times \mathbb{P}^{1}$ be curves of types $(0,1),(1,0),(1,1)$, and $(1,1)$, respectively. Suppose that there exists a unique point where $Z_{1}, Z_{3}$, and $Z_{4}$ intersect transversally and that outside of this point of triple intersection the $Z_{i}$ are in general position. Then there exists a proper Zariski-closed subset $Y \subset \mathbb{P}^{1} \times \mathbb{P}^{1}$, independent of $k$ and $S$, such that for any set $R$ of $S$-integral points on $\mathbb{P}^{1} \times \mathbb{P}^{1} \backslash \cup_{i=1}^{4} Z_{i}$ the set $R \backslash Y$ is finite.

Remark. The assumptions of this theorem do not quite satisfy the assumptions of Corollary 1.2 in [6] (with $D_{1}=Z_{1} \cup Z_{2}, D_{2}=Z_{3}$, and $D_{3}=Z_{4}$ in their notation). However, the proof in [6] shows that instead of assuming $D_{1}, D_{2}$, and $D_{3}$ are irreducible, it is sufficient that the strict transforms of $D_{1}, D_{2}$, and $D_{3}$ in the blow-up at the point of triple intersection be linearly equivalent to irreducible effective divisors, which certainly occurs in our situation.

Let $P$ be the point of triple intersection in Theorem 13. It is easily seen that the set $Y$ in Theorem 13 can be taken to consist of curves of type $(1,0)$ and $(0,1)$, a curve of type $(1,1)$ tangent to $Z_{3}$ and passing through $P$ and $Z_{2} \cap Z_{4}$, and a curve of type $(1,1)$ tangent to $Z_{4}$ and passing through $P$ and $Z_{2} \cap Z_{3}$. Using Theorem 7 to translate this into arithmetic, we obtain

Corollary 14. Let $L_{1}=a_{1} t+a_{0}, L_{2}=b_{1} t+b_{0}$, and $L_{3}=c_{1} t+c_{0}$ with $L_{1}, L_{2}, L_{3} \in$ $k[t], L_{1} / L_{2}$ nonconstant, and $a_{1}, b_{1}, c_{1} \neq 0$. All but finitely many nontrivial solutions to

$$
L_{1}(t) u+L_{2}(t) v=L_{3}(t) \quad \text { in } t \in \mathcal{O}_{k, S}, \quad u, v \in \mathcal{O}_{k, S}^{*},
$$

are parametrized by the following four families:

$$
\begin{aligned}
& t=\frac{\left(a_{0} b_{1}-a_{1} b_{0}\right) \eta}{b_{1} c_{1}}-\frac{c_{0}}{c_{1}}, \quad u=\eta, \quad v=-\frac{a_{1} \eta}{b_{1}}, \quad \eta \in \mathcal{O}_{k, S}^{*} \\
& t=\frac{a_{1} c_{0}-a_{0} c_{1}-a_{1} b_{0} \eta}{a_{1} b_{1} \eta}, \quad u=\frac{c_{1}}{a_{1}}, \quad v=\eta, \quad \eta \in \mathcal{O}_{k, S}^{*} \\
& t=\frac{b_{1} c_{0}-b_{0} c_{1}-a_{0} b_{1} \eta}{a_{1} b_{1} \eta}, \quad u=\eta, \quad v=\frac{c_{1}}{b_{1}}, \quad \eta \in \mathcal{O}_{k, S}^{*} \\
& t \in \mathcal{O}_{k, S}, \quad u=\frac{b_{0} c_{1}-b_{1} c_{0}}{a_{1} b_{0}-a_{0} b_{1}}, \quad v=\frac{a_{0} c_{1}-a_{1} c_{0}}{a_{0} b_{1}-a_{1} b_{0}}
\end{aligned}
$$

Corollary 14 was already proven, implicitly, in [6, Remark 4.2].

\section{Acknowledgments}

I would like to thank Pietro Corvaja and Umberto Zannier, without whom this paper would not have been written. The ideas involved originated in conversations amongst the three of us while attending the program on Diophantine Geometry in 2005 at the De Giorgi Center in Pisa, Italy.

\section{References}

[1] F. Beukers and H. P. Schlickewei, The equation $x+y=1$ in finitely generated groups, Acta Arith. 78 (1996), no. 2, 189-199.

[2] E. Bombieri, J. Mueller, and M. Poe, The unit equation and the cluster principle, Acta Arith. 79 (1997), no. 4, 361-389. 
[3] P. Corvaja and U. Zannier, A subspace theorem approach to integral points on curves, C. R. Math. Acad. Sci. Paris 334 (2002), no. 4, 267-271.

[4] - On the number of integral points on algebraic curves, J. Reine Angew. Math. 565 (2003) $27-42$.

[5] - On integral points on surfaces, Ann. of Math. 160 (2004), no. 2, 705-726.

[6] - On the integral points on certain surfaces, Int. Math. Res. Not. (2006) Art. ID 98623, 20 pages.

[7] J.-H. Evertse, On equations in S-units and the Thue-Mahler equation, Invent. Math. 75 (1984), no. $3,561-584$.

[8] - On sums of S-units and linear recurrences, Compositio Math. 53 (1984), no. 2, 225-244.

[9] - An improvement of the quantitative subspace theorem, Compositio Math. 101 (1996), no. 3, 225-311.

[10] J.-H. Evertse and K. Györy, On the numbers of solutions of weighted unit equations, Compositio Math. 66 (1988), no. 3, 329-354.

[11] J.-H. Evertse, K. Györy, C. L. Stewart, and R. Tijdeman, On S-unit equations in two unknowns, Invent. Math. 92 (1988), no. 3, 461-477.

[12] K. Győry, On the number of solutions of linear equations in units of an algebraic number field, Comment. Math. Helv. 54 (1979), no. 4, 583-600.

[13] R. Hartshorne, Algebraic geometry, Springer-Verlag, New York (1977), ISBN 0-387-90244-9. Graduate Texts in Mathematics, No. 52.

[14] S. Lang, Integral points on curves, Inst. Hautes Études Sci. Publ. Math. (1960), no. 6, 27-43.

[15] A. Levin, Generalizations of Siegel's and Picard's Theorems, To appear in Annals of Mathematics.

[16] A. J. van der Poorten and H. P. Schlickewei, The growth condition for recurrence sequences, Macquarie Univ. Math. Rep. 82-0041 (1982)

[17] — Additive relations in fields, J. Austral. Math. Soc. Ser. A 51 (1991), no. 1, 154-170.

Department of Mathematics, Brown University, 151 Thayer Street, Providence, Ri

E-mail address: adlevin@math.brown.edu 